\title{
Bemerkungen zur Textgestaltung
}

Im Jahre 1962 wurde der 1. Teil des Reisejournals D. G. Messerschmidts vorgelegt, der - neben einer Einleitung, in der die Bedeutung der Forschungsreise Messerschmidts durch Sibirien 1720 bis 1727 gewürdigt wurde - die Tagebuchaufzeichnungen aus den Jahren 1721 und 1722 enthielt. Diese wurden dem ursprünglichen Editionsplan gemäß im vollen Wortlaut wiedergegeben und gewährten so Einblick in alle Einzelheiten der Reisevorbereitungen und des Reiseverlaufs, der Gespräche und des Briefwechsels sowie der Gedanken des Forschungsreisenden zu den verschiedensten Erscheinungen seiner Umwelt.

Es hat sich als notwendig erwiesen, die ursprünglich auf 10 Bände berechnete Ausgabe wesentlich zu kürzen. Dies soll durch Verzicht auf den Abdruck anderer Arbeiten Messerschmidts und einen verknappten Kommentar erreicht werden, wirkt sich aber auch auf die Wiedergabe der Tagebuchaufzeichnungen aus, die nunmehr nur noch insgesamt 4 von ursprünglich 5 Bänden bei einem festgelegten Umfang umfassen werden.

Die vom Kollegium für die Herausgabe der Tagebücher Messerschmidts erarbeiteten Richtlinien für die Kürzung gehen davon aus, daß der wissenschaftliche Wert der Tagebuchaufzeichnungen nicht geschmälert werden darf und die gekürzte Ausgabe wissenschaftlich vertretbar sein muß. Deshalb bleiben alle wissenschaftlich interessanten Beobachtungen sowie die wissenschaftlichen Exkurse auf den verschiedenen Wissensgebieten (Zoologie, Botanik, Mineralogie, Geologie, Geographie, Ethnographie, Sprachwissenschaft usw.) erhalten. Lediglich lange lateinische Beschreibungen von Sektionsbefunden werden durch kurze deutsche Resümees ersetzt. Bei der Aufzählung von Pflanzen (besonders Heilkräutern), die von Messerschmidt und seinen Begleitern gesammelt wurden, werden Wiederholungen vermieden. Das gleiche gilt bei den von Messerschmidts Gehilfen geschossenen Vögeln.

Stark gekürzt wurde die Schilderung des Tagesablaufs, insbesondere im Winterquartier. Die Orientierung über den Reiseweg bleibt durch die Untergliederung in Reiseabschnitte, die Kartenskizze der Reiseroute sowie durch kurze Übersichten am Beginn jedes Reiseabschnitts bzw. der einzelnen Tage gegeben. In diesen Übersichten werden alle von Messerschmidt im Reisejournal genannten Orte und Flüsse angeführt. Kommt bei Orten, die an der Mündung von Flüssen liegen, diese Tatsache bereits im Ortsnamen durch Zusammensetzung von „Ust'-“ [-münde] mit dem Namen des Flusses zum Ausdruck (z. B. Ust'-Kija), wird der Flußname (Kija) nicht besonders erwähnt. Die täglichen Wetterbeobachtungen werden nicht gebracht. Ebenso entfallen die meisten Berichte über den Verkehr mit den Behörden, über Beschäftigung und Verhalten der Untergebenen, die Wiedergabe des Wortlauts oder des Inhalts empfangener und abgesandter Briefe, die Darstellung von 
Gesprächen, soweit sie nicht wissenschaftliche Ausführungen enthalten oder für die Reise von unmittelbarer Bedeutung sind, die Zusammensetzung vieler von Messerschmidt bei seiner ärztlichen Praxis verordneter Medikamente sowie alle Reflexionen allgemeiner Art.

Die im Teil 1 gegebene „Übersicht über die Tagebuchaufzeichnungen“ wird nicht fortgesetzt; da jetzt fast nur noch die wissenschaftlichen Ausführungen gedruckt werden, ist sie für die Erschließung des Textes nicht mehr im gleichem Maße erforderlich. Personen und geographisches Register folgen, durch ein Sachregister ergänzt, der Veröffentlichung des ganzen Reisejournals, nicht mehr den einzelnen Teilen.

Im übrigen gelten die in Teil 1 erläuterten Editionsprinzipien weiter. Bei den Vokabularen der sibirischen Sprachen wird jedoch - soweit dies eindeutig feststellbar ist - die von Messerschmidt gegebene Akzentsetzung beibehalten. Auch bei den gekürzt wiedergegebenen Textpartien ist der Wortlaut der Tagebuchaufzeichnungen Messerschmidts treu bewahrt. Kürzungen innerhalb einer zusammenhängenden Stelle (eines Absatzes) sind durch ... bezeichnet. Diese Kennzeichnung entfällt, wenn nur ein hinweisendes Wort (z. B.: hier, dann) durch eine Orts- oder Zeitangabe in [...] ersetzt wurde.

Es sei darauf verwiesen, daß sich schon frühere Generationen mit der Frage einer gekürzten Wiedergabe der Tagebuchaufzeichnungen Messerschmidts beschäftigt haben. So schrieb P. S. Pallas am 28. 5./8. 6. 1769 aus Samara an den Ständigen Sekretar der Petersburger Akademie, J. A. Euler ${ }^{1}$ : „... Des Messerschmidtischen Journals will ich, obwohl ungern, entbehren. Ew. Wohlgeboren verlangen, da $B$ ich meine Gedanken von der Ausgabe desselben der Akademie eröffnen soll. Ich habe es, soviel mir in dem letzten Monate vor meiner Abreise die Kürze der Zeit erlaubte, durchgesehen. Allerdings enthält es eine Menge von wichtigen Nachrichten, ja viel mehr Nützliches als der größte Teil der Gmelinschen Reisebeschreibung. Allein es erfordert eine geschickte Feder und viel Geduld, um einen guten und zum Drucke tüchtigen Auszug daraus zu verfertigen ..." Möge der hier unternommene Versuch als geglückt angesprochen werden können!

1 Akademie-Archiv Leningrad, F. 1, op. 3, Bd. 53, No. 39; Kopie (in einigen Formulierungen vom Original abweichend) ebd., F. 1, op. 3, Bd. 51, Abt. II, No. 62. 
